\section{T. Dongmo}

\section{Pouilles-Duplaix ${ }^{1}$}

\section{Picard ${ }^{2}$}

C. $\mathrm{Mbi}^{3}$

M. De Reviers ${ }^{2}$

\section{Utilisation du tourteau de coton dans l'alimentation des volailles. II. Effets du gossypol sur les paramètres de la reproduction}

DONGMO (T.), POUILlES-DUPLAIX (M.), PICARD (M.), MBI (C), DE REVIERS (M.). Utilisation du tourteau de coton dans l'alimentation des volailles. II. Effet du gossypol sur les paramètres de la reproduction. Revue Élev. Méd. vét. Pays trop., 1993, 46 (4) : 621-630

Trois expériences mesurent les effets du gossypol, administré par voie alimentaire, sur la reproduction des coqs et de poules adultes de souches ponte ou chair. Six cent cinquante grammes d'acétate de gossypol ont d'ahord été extraits et cristallisés à partir de $80 \mathrm{~kg}$ de soapstock de coton. Le produit obtenu titrait 47,86 p. 100 de gossypol libre et 61,19 p. 100 de gossypol total. Des aliments équilibrés, dépourvus de tourteau de coton ont été complémentés avec des doses croissantes de $0,250,400$ et $800 \mathrm{ppm}$ de gossypol libre et distribués à des coqs et à des poules reproductrices. Le premier essai a porté sur 128 poules et 45 coqs pubères; il ne montre aucun effet de doses alimentaires inférieures ou égales à $\mathbf{4 0 0} \mathbf{p p m}$ de gossypol libre sur la consommation alimentaire, la ponte, la production et la motilité du sperme, la fécondité et l'éclosabilité des eufs incubés. Les deux essais suivants ont servi à tester l'effet d'une concentration alimentaire de $800 \mathrm{ppm}$ de gossypol libre sur respectivement 10 cogs reproducteurs de souche chair âgés de 82 semaines et rationnés et 12 coqs reproducteurs de souche ponte âgés de 29 semaines nourris ad libitum. A cette dose (environ $20 \mathrm{mg} / \mathrm{kg} \mathrm{PV/j}$ ) le gossypol libre a diminué la consommation alimentaire, le poids des animaux et des testicules des coqs de souche ponte et fait augmenter la proportion de spermatozoïdes anormaux dans les éjaculats des coqs de souche chair. Pour cette concentration on a observé une couleur anormale brun verdâtre des foies provenant de coqs ayant consommé les régimes complémentés en gossypol. Compte tenu des concentrations du tourteau de coton en gossypol (en général inférieures à $250 \mathrm{ppm}$ ), il semble peu vraisemblable que le cossypol libre de ce tourteau soit directement responsable des troubles de la reproduction observés chez les volailles qui en consomment.

Mots clés : Volaille - Coq - Poule pondeuse - Poulet de chair - Alimentation -Tourteau de coton - Gossypol - Reproduction - Performance de ponte - Éclosabilité - Sperme - Poids.

\section{INTRODUCTION}

Le tourteau de coton (TC), utilisé comme principale source de protéines à la place du tourteau de soja dans l'alimentation des poules reproductrices, freine leur croissance et réduit le nombre et la taille des œufs pondus, même lorsque l'aliment est correctement supplémenté en lysine et sulfate de fer (10). Toutefois la fertilité et l'éclosabilité des œufs produits ne sont pas significativement

1. Institut de recherches zootechniques, BP 1457, Yaoundé, Cameroun.

2. Institut national de la recherche agronomique (INRA), Station de Recherches avicoles, 37380 Nouzilly, France.

3. Institut de recherches médicales et d'études des plantes médicinales, BP 193, Yaoundé, Cameroun.

Reçu le 2.3.1993, accepté le 20.4.1993. diminuées par la consommation du TC dans nos conditions expérimentales, alors que le gossypol ajouté seul à une ration ne contenant pas de TC réduit la fertilité des rats (27) et des singes (14) qui en consomment. Chez les volailles, AKANBI et al. (3) observent une réduction de la fertilité pour des concentrations alimentaires de gossypo supérieures ou égales à 1000 ppm tandis que SAVILLE et al. (23) observent un effet similaire pour des doses dix fois inférieures. Il existe donc une incertitude sur les doses de gossypol alimentaire susceptibles d'affecter les facultés de reproduction des volailles.

Les variétés de coton "glandless", c'est-à-dire dépourvues de gossypol semblent bien utilisées par les poulets en croissance (28). Dès lors, quels sont les effets nocifs spécifiques du gossypol sur la croissance et la reproduction de ces animaux ? Beaucoup d'expériences publiées sur le TC dissocient mal l'effet du gossypol de celui des autres facteurs alimentaires ou toxiques pouvant agir simultanément sur les performances et l'état sanitaire des animaux. Le but du travail que nous rapportons ici est de tenter d'identifier et de quantifier les effets éventuels du seul gossypol sur la reproduction des coqs et poules reproducteurs, en dehors de son support alimentaire habituel : le tourteau de coton (tableau I).

\section{MATÉRIEL ET MÉTHODES}

\section{Extraction et purification du gossypol}

Les graines de coton contiennent en moyenne de 0,4 à 1,4 p. 100 de gossypol (4) ; le tourteau est beaucoup moins riche (environ 0,5 à 1,5 p. 100 de gossypol) que le "soapstock"* (4 à 6 p. 100).

Un échantillon de $1 \mathrm{~kg}$ de "soapstock" en provenance de l'huilerie de Garoua au Cameroun est placé dans un balIon de deux litres et lavé par agitation pendant deux minutes avec de l'hexane -SIGMA A6283- (300 ml $/ \mathrm{kg}$ de soapstock). Le mélange est alors transvasé dans une ampoule à décanter et la fraction lourde est conservée. De cette fraction, $500 \mathrm{~g}$ sont introduits dans un ballon de deux litres avec $500 \mathrm{~g}$ d'acide méta-phosphorique. ALDRICH 31027 - (dilué à $0,4 \mathrm{M}$ ) et $500 \mathrm{~g}$ de butanone SIGMA M2886- selon la méthode décrite par PONS et al. (21). Ce mélange est chauffé à $50^{\circ} \mathrm{C}$ et agité pendant 90

\footnotetext{
* Soapstock : résidu de neutralisation issu de l'action de la soude sur les acides gras libres contenus dans l'huile brute.
} 
TABLEAU I Thèmes des essais.

\begin{tabular}{|c|c|c|c|c|}
\hline Thème étudié & $\begin{array}{l}\text { Titre d'essai } \\
\text { Essai 1. Effets de } 250 \\
\text { et } 400 \text { ppm de } \\
\text { gossypol sur les coqs } \\
\text { et les poules }\end{array}$ & $\begin{array}{l}\text { Nombre } \\
\text { d'animaux }\end{array}$ & $\begin{array}{l}\text { Durée essai } \\
9 \text { semaines }\end{array}$ & $\begin{array}{l}\text { Paramètres étudiés } \\
\text { — consommation alimentaire } \\
\text { — intensité de ponte } \\
\text { - poids des œufs } \\
\text { - fertilité } \\
\text { - éclosivité } \\
\text { - qualité du sperme }\end{array}$ \\
\hline \multirow[t]{2}{*}{$\begin{array}{l}\text { Effets du } \\
\text { gossypol } \\
\text { l'aliment sur les } \\
\text { paramètres de } \\
\text { la reproduction }\end{array}$} & $\begin{array}{l}\text { Essai } 2 \text { - Effets de } 800 \text { ppm } \\
\text { de gossypol sur la production } \\
\text { de spermatozoïdes de coqs } \\
\text { de type chair rationnés. }\end{array}$ & 10 coqs & 6 semaines & $\begin{array}{l}\text { - consommation alimentaire, } \\
\text { - qualité du sperme } \\
\text { + motilité } \\
\text { + concentration des éjaculats } \\
\text { + poids des éjaculats } \\
\text { + morphologie des spermatozoïdes } \\
\text { - poids des } \\
\text { organes internes }\end{array}$ \\
\hline & $\begin{array}{l}\text { Essai } 3 \text {. Effets de } 800 \mathrm{ppm} \text { de } \\
\text { gossypol sur la consommation } \\
\text { alimentaire de coqs } \\
\text { reproducteurs ponte nourris } \\
\text { ad libitum. }\end{array}$ & 12 coqs & 7 semaines & $\begin{array}{l}\text { - consommation alimentaire, } \\
\text { - poids des organes } \\
\text { - contrôles histologiques }\end{array}$ \\
\hline
\end{tabular}

minutes. La température et le temps ont été choisis pour maximiser le rendement d'extraction (à $50^{\circ} \mathrm{C}$ le gossypol se lie moins aux autres molécules (9)). Après refroidissement à température ambiante le mélange est introduit dans une ampoule à décanter et la phase légère est conservée. Cette fraction est concentrée au tiers du volume par évaporation sous vide et chauffage à $50^{\circ} \mathrm{C}$. Après mesure du volume restant, la fraction est mélangée avec un tiers de volume d'acide acétique cristallisable -SIGMA A6283- dans un erlenmeyer de deux litres. Le mélange obtenu est agité puis laissé pendant 48 heures à la température du laboratoire (environ $25^{\circ} \mathrm{C}$ ), avant d'être filtré sous vide sur papier filtre. Les cristaux d'acétate de gossypol sont lavés sur papier filtre à l'hexane jusqu'à obtention d'une couleur jaune pâle puis séchés à l'étuve à $40^{\circ} \mathrm{C}$ pendant 24 heures. Les cristaux secs d'acétate de gossypol sont pesés, placés dans un bécher puis dissous avec $6 \mathrm{ml}$ de butanone par gramme de cristaux. Le gossypol est recristallisé sous la forme d'acétate par addition de $2 \mathrm{ml}$ d'acide acétique cristallisable par gramme. Les cristaux sont immédiatement filtrés sous vide et séchés à l'étuve $\left(40^{\circ} \mathrm{C}\right.$ pendant 24 heures). Le rendement moyen obtenu est de $650 \mathrm{~g}$ d'acétate de gossypol brut pour 80 $\mathrm{kg}$ de soapstocks traités.

L'analyse de l'acétate de gossypol brut obtenu, réalisée par HPLC, indique une teneur de 61.19 p. 100 de gossypol total dont 47,86 p. 100 sous forme libre.

\section{Essai 1 \\ Effets de concentrations variables de gossypol ( 250 et $400 \mathrm{ppm}$ ) incorporé dans le régime alimentaire chez des coqs et des poules reproducteurs}

La teneur en gossypol des aliments pour volailles fabriqués au Cameroun avec du tourteau de coton est en moyenne de $150 \mathrm{ppm}$. Les doses testées ici (250 et 400 ppm) sont donc supérieures aux concentrations maximales généralement rencontrées $(20,22)$.

\section{Animaux}

Cent vingt huit poules pondeuses semi-lourdes de souche "Lohmann" âgées de 21 semaines sont réparties dans 64 cages de 2 poules dans deux batteries californiennes identiques placées en bâtiment ouvert.

Quarante-cinq coqs de souche chair "Arbor Acres" âgés de 18 semaines sont logés individuellement dans des cages situées dans le même bâtiment que les poules. Tous les animaux ont reçu pendant leur croissance des aliments à base de maïs/soja sans tourteau de coton ni gossypol.

\section{Aliments et répartition des animaux}

Deux régimes de base sans tourteau de coton ni gossypol sont formulés pour satisfaire les besoins nutritionnels 
des poules (nourries ad libitum) et des coqs (sur la base d'un rationnement quantitatif à $115 \mathrm{~g}$ d'aliment par animal et par jour) (tableau II). Le régime de ponte est supplémenté avec 0, 250 ou 400 ppm de gossypol libre en tenant compte des résultats analytiques $(47,86$ p. 100 de gossypol libre dans l'acétate brut). Le régime destiné aux coqs est supplémenté avec 0 ou 400 ppm de gossypol libre. A partir de 21 semaines d'âge, chacun des 2 aliments contenant du gossypol est distribué ad libitum à 32 poules (16 cages de 2 ) alors que le régime témoin sans gossypol est distribué ad libitum à 64 poules (32 cages de 2). A partir de 22 semaines d'âge, les aliments contenant respectivement 0 et 400 ppm de gossypol sont distribués $(115 \mathrm{~g} / \mathrm{j})$ à 22 et 23 coqs. La durée totale de l'expérience est de 9 semaines. Pendant cette période, tous les animaux reçoivent un éclairement journalier de $16 \mathrm{~h}$. Afin d'estimer l'incidence éventuelle du gossypol sur la fertilité des deux sexes, les poules témoins sont inséminées par du sperme provenant des coqs témoins ou recevant du gossypol, alors que les poules consommant du gossypol sont inséminées par du sperme produit par les seuls coqs témoins.

La répartition des poules suit un dispositif en 4 blocs randomisés. Chaque bloc, composé de 16 cages voisines, est subdivisé en 4 groupes de 4 cages. Chacun de ces groupes reçoit un traitement différent : aliment témoin (insémination par coqs témoins), aliment témoin (insémination par coqs consommant du gossypol), aliment 250 ppm de gossypol ou aliment 400 ppm de gossypol (insémination par coqs témoins).

\section{Critères retenus}

La consommation alimentaire et la production d'cufs (nombre et poids) sont enregistrées pendant toute l'expérience. Les coqs sont entraînés à la production de sperme par massage dorso-lombaire trois fois par semaine (6) puis le sperme de chaque coq est récolté et analysé quotidiennement pendant 5 jours consécutifs au cours de la 29 e semaine d'âge (soit après 6 semaines de distribution des aliments expérimentaux). Le volume des éjaculats est mesuré avec une pipette graduée (précision : $0,01 \mathrm{ml}$ ), leur concentration estimée avec un photomètre préalablement étalonné pour le sperme de coq (IMV, l'Aigle, France) et sa motilité massale est évaluée subjectivement au microscope (19).

Après sept semaines de consommation des régimes expérimentaux pour les coqs et quatre semaines pour les poules, trois inséminations artificielles sont réalisées, les deux premières à $24 \mathrm{~h}$ d'intervalle et la troisième six jours plus tard. Chaque insémination est pratiquée en sperme pur avec 60 millions de spermatozoïdes issus des éjaculats mélangés des coqs d'un mème traitement. Les œufs pondus pendant les 15 jours après la seconde insémination sont mis en incubation deux fois par semaine puis mirés aux septième et dix-huitième jours ,pour estimer leur fertilité ainsi que la survie embryonnaire.
Tous les animaux sont pesés en début et en fin d'expérience (à 21 et 28 semaines d'âge pour les poules, 22 et 31 semaines d'âge pour les coqs). Chez les poules la consommation alimentaire, l'intensité de ponte, le poids des œufs, la masse d'œuf exportée, l'indice de consommation et l'index de coquille (calculé selon la formule de SCOTT et MUELLER (24)) sont mesurés pendant une période de 31 jours de 23 à 28 semaines d'âge, soit après une période d'adaptation aux régimes expérimentaux de 12 jours.

Chez les mâles le volume de sperme, sa concentration en spermatozoïdes et leur motilité sont estimés individuellement. Enfin, la fertilité et l'éclosivité des œufs sont analysées à partir des moyennes des mesures faites dans chaque lot expérimental (un coq ou un groupe de 8 poules).

\section{Analyse statistique}

Les données brutes sont comparées par analyse de la variance (dispositif en blocs) en utilisant le logiciel Systat. Dans le cas des proportions (pourcentages de fertilité, d'éclosivité) les données sont transformées en arcsinus racine carrée avant analyse. Les moyennes sont comparées par le test de NEWMAN et KEULS.

\section{Essais 2 et 3}

\section{Effets de doses massives de gossypol (800 ppm) chez les coqs reproducteurs}

La dose de gossypol alimentaire affectant significativement la reproduction chez le rat est de $20 \mathrm{mg} / \mathrm{kg}$ de $\mathrm{PV} / \mathrm{j}$ $(12,26)$. La concentration de 800 ppm est choisie pour atteindre cette dose chez les coqs. L'essai 2 concerne des reproducteurs lourds de type chair rationnés quantitativement, alors que l'essai 3 se rapporte à des coqs reproducteurs de type ponte nourris ad libitum; aucun de ces coqs n'a subi de traitement particulier avant l'expérience.

Pour l'essai 2, on a utilisé 10 coqs de souche label chair "T55" âgés de 82 semaines et logés individuellement dans les cages d'une cellule conditionnée en lumière (14 $\mathrm{h} / \mathrm{j})$ et température $\left(20-23^{\circ} \mathrm{C}\right)$. L'essai 3 a été réalisé sur 12 coqs de souche ponte "Isabrown" âgés de 29 semaines et placés en cages individuelles dans les mêmes conditions d'environnement que les coqs de l'essai 2.

\section{Aliments et répartition des animaux}

Dans l'essai 2, un régime de base équilibré par rapport aux besoins nutritionnels des animaux (tableau II) est supplémenté ou non avec 800 ppm de gossypol libre (soit 1672 ppm d'acétate de gossypol brut), alors que dans l'essai 3 la même supplémentation est réalisée 
TABLEAU II Composition et caractéristiques calculées des régimes alimentaires des 3 essais.

\begin{tabular}{|c|c|c|c|c|}
\hline & \multicolumn{2}{|c|}{ Essai 1} & \multirow{2}{*}{$\frac{\text { Essai } 2}{\text { Régime coq }}$} & \multirow{2}{*}{$\frac{\text { Essai } 3}{\text { Régime ponte }}$} \\
\hline & Régime coq & Régime poule & & \\
\hline $\begin{array}{l}\text { Maïs } \\
\text { Blé } \\
\text { Tourteau de soja } \\
\text { Tourteau de tournesol } \\
\text { Tourteau de maïs } \\
\text { Drèches séchées } \\
\text { Son de riz } \\
\text { Farine de sang } \\
\text { Farine de viande } 60 \\
\text { Gluten de maïs } \\
\text { Huile de colza } \\
\text { Huile de palme } \\
\text { Graine } 15 \\
\text { Concentré minéral } \\
\text { Coquillage } \\
\text { Prémélange } \\
\text { Lysine HCl } \\
\text { DL-Méthionine } \\
\text { CaCO } \\
\text { Phosphate bicalcique } \\
\text { Coccidiostat }\end{array}$ & $\begin{array}{l}\frac{65}{10,7} \\
- \\
12,28 \\
6 \\
0 \\
- \\
- \\
- \\
- \\
- \\
4,5 \\
0,4 \\
1 \\
0,12 \\
- \\
- \\
- \\
100\end{array}$ & $\begin{array}{c}56,3 \\
\overline{20,4} \\
- \\
- \\
- \\
4,7 \\
- \\
- \\
1 \\
- \\
7,8 \\
1 \\
\overline{0}, 1 \\
- \\
- \\
100\end{array}$ & $\begin{array}{l}33 \\
49,5 \\
6 \\
- \\
- \\
- \\
- \\
4,2 \\
2,0 \\
- \\
-1,5 \\
- \\
-0,9 \\
0 \\
0,015 \\
2,0 \\
0,8 \\
0,075 \\
99,99\end{array}$ & $\begin{array}{l}40,5 \\
25,63 \\
17,2 \\
1,25 \\
- \\
- \\
- \\
2,85 \\
- \\
1,55 \\
- \\
- \\
-1,45 \\
\overline{-} \\
0,09 \\
1,19 \\
\overline{1}, 29 \\
100\end{array}$ \\
\hline \multicolumn{5}{|c|}{ Caractéristiques calculées } \\
\hline $\begin{array}{l}\text { Energie métabolisable kcal } / \mathrm{kg} \\
\text { Protéines brutes (p. 100) } \\
\text { Lysine (p. 100) } \\
\text { Méthionine (p. 100) } \\
\text { Phosphore disponible (p. 100) } \\
\text { Calcium (p. 100) }\end{array}$ & $\begin{array}{l}2710 \\
14,9 \\
0,7 \\
0,32 \\
0,47 \\
1\end{array}$ & $\begin{array}{l}2721 \\
17,9 \\
0,98 \\
0,42 \\
0,4 \\
3,65\end{array}$ & $\begin{array}{c}2880 \\
14,6 \\
0,56 \\
0,26 \\
0,6 \\
1,3\end{array}$ & $\begin{array}{r}2700 \\
17,26 \\
0,83 \\
0,36 \\
0,38 \\
3,40\end{array}$ \\
\hline
\end{tabular}

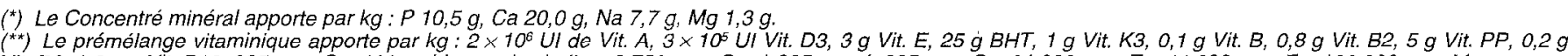
Vit. B6, t,6 mg Vit. B12, $335 \mathrm{ppm} \mathrm{Co,} 100 \mathrm{~g}$ chlorure de choline, $8750 \mathrm{ppm} \mathrm{Cu}, 1225 \mathrm{ppm} \mathrm{I,} 225 \mathrm{ppm}$ Se, $84000 \mathrm{ppm} \mathrm{Zn}, 44000 \mathrm{ppm}$ Fe, $106000 \mathrm{ppm} \mathrm{Mn}$.

dans le régime "ponte" que recevaient les coqs depuis plusieurs semaines (tableau II). Dans chaque essai, une moitié des coqs est alimentée avec le régime approprié sans gossypol afin de constituer les lots témoins. La durée des protocoles expérimentaux est de six semaines dans l'essai 2 et de sept dans l'essai 3. Les coqs de l'essai 2 sont rationnés à $100 \mathrm{~g}$ d'aliment par jour alors que les coqs de l'essai 3 sont nourris ad libitum. La consommation d'aliment est mesurée chaque semaine. Les animaux sont pesés en début et fin d'expérience puis sacrifiés et autopsiés. Les poids et l'aspect de plusieurs organes sont étudiés (foie, testicules, cœur, rate pour les deux essais et gras abdominal et reins dans le cas de l'essai 3). Au cours de l'essai 2, le sperme des coqs est rćcolté trois fois par semaine. Quatre éjaculats par coq sont analysés pendant les quatre derniers jours de l'expérience. Le poids de chaque éjaculat $( \pm 0,01 \mathrm{~g})$, la concentration et la motilité du sperme sont évalués en utilisant les méthodes décrites pour l'essai 1. Un test de coloration à l'éosine-nigrosine permet d'estimer la proportion de spermatozoïdes vivants, malformés ou morts (16). A l'issue de l'essai 3, un prélèvement histologique est réalisé sur les foies, les testicules et la rate des coqs des deux groupes. Les tissus sont fixés dans du liquide de Bouin et examinés en microscopie optique après coloration à l'hémalun-éosine-safran (HES).

\section{Analyse statistique}

Les comparaisons' de moyennes entre régime témoin et aliment contenant 800 ppm de gossypol sont effectuées par un test $t$ après transformation des pourcentages en arcsinus racine carrée (spermatozoïdes morts, vivants ou malformés). 
TABLEAU III Essai 1. Effet du gossypol sur la reproduction. Résultats moyens de la période 23-28 semaines d'âge.

\begin{tabular}{|c|c|c|c|c|c|}
\hline $\begin{array}{l}\text { Régimes poules } \\
\text { (Nombre de poules) }\end{array}$ & $\begin{array}{l}\text { Témoin } \\
\text { (32) }\end{array}$ & $\begin{array}{l}\text { Témoin } \\
\text { (32) }\end{array}$ & $\begin{array}{l}250 \mathrm{ppm} \\
(32)\end{array}$ & $\begin{array}{l}400 \mathrm{ppm} \\
(32)\end{array}$ & \multirow{2}{*}{$\mathrm{SEM}^{1}$} \\
\hline Régimes coqs & Témoin & $400 \mathrm{ppm}$ & Témoin & (Témoin) & \\
\hline Consommation $(\mathrm{g} / \mathrm{p} / \mathrm{j})$ & 110,9 & 111,6 & 110,4 & 111,8 & 3,19 \\
\hline Intensité de ponte (p. 100) & 79,9 & 84,3 & 82,2 & 80,8 & 3,2 \\
\hline Poids moyen des œufs $(\mathrm{g})$ & 52,4 & 52,7 & 54,0 & 53,4 & 0,72 \\
\hline Masse d'œuf produite (g/j/p) & 41,9 & 44,5 & 44,5 & 43,2 & 2,10 \\
\hline Indice de consommation & 2,65 & 2,54 & 2,51 & 2,62 & 0,14 \\
\hline $\begin{array}{l}\text { Gain de Poids } \\
\text { (g/poule) }\end{array}$ & 150,8 & 129,8 & 156,3 & 176,5 & 17,0 \\
\hline $\begin{array}{l}\text { Index de coquille } \\
\left(\mathrm{dg} / \mathrm{cm}^{2}\right)\end{array}$ & 7,68 & 7,71 & 7,48 & 7,75 & 0,09 \\
\hline Nombre d'œufs mis en incubation & 344 & 390 & 377 & 411 & \\
\hline Fertilité (p. 100) & 90,9 & 93,3 & 92,7 & 93,5 & 0,16 \\
\hline $\begin{array}{l}\text { Eclosabilité vraie } \\
\text { (éclos/fertiles) (p. 100) }\end{array}$ & 61,3 & 68,1 & 67 & 70,8 & 0,27 \\
\hline $\begin{array}{l}\text { Eclosivité totale } \\
\text { (éclos/incubés) (p. 100) }\end{array}$ & 55,6 & 63,5 & 62,1 & 66,1 & 0,32 \\
\hline
\end{tabular}

(1) SEM : Erreur standard de la moyenne. Aucune différence significative (au seuil de 5 p. 100) n'est apparue dans cet essai pour chacun des critères analysés.

\section{RÉSULTATS}

\section{Essai 1}

Effets de 250 et 400 ppm de gossypol dans le régime alimentaire sur la reproduction des coqs et des poules

Aucune mortalité anormale pouvant être attribuée à un traitement n'est notée au cours de la période expérimentale. Chez les poules les résultats moyens de consommation alimentaire, de taux de ponte et de caractéristiques des œufs, de variations du poids vif ne diffèrent pas significativement $(P>0,05)$ entre les traitements étudiés (tableau III).

L'addition de 250 ou 400 ppm de gossypol dans le régime ne réduit pas la consommation alimentaire quotidienne qui est comparable $(110,4$ et $111,8 \mathrm{~g} / \mathrm{j})$ à celle des poules consommant un aliment témoin (110,9 et 111,6 $\mathrm{g} / \mathrm{j}$ ). II en est de même de la masse d'œuf moyenne exportée qui varie de $41,9 \mathrm{~g} / \mathrm{j}$ pour l'un des groupes témoins à $44,5 \mathrm{~g} / \mathrm{j}$ pour les animaux recevant un régime contenant 250 ppm de gossypol. L'indice de consommation varie peu d'un traitement à l'autre (2,51 pour 250 ppm de gossypol à 2,65 pour le groupe témoin).

Les résultats moyens de fertilité et d'éclosivité sont comparables entre les groupes témoins et expérimentaux (tableau III). De même, les caractéristiques moyennes du spérme produit par les coqs au cours de cette expérience (tableau IV) ne diffèrent pas significativement $(P<0,05)$ selon que les coqs ont consommé ou non du gossypol (environ $13 \mathrm{mg} / \mathrm{kg} \mathrm{PV} / \mathrm{j}$ ).

\section{Essai 2}

Effets de rations alimentaires contenant 800 ppm de gossypol sur la production de spermatozoïdes de coqs reproducteurs de type chair

La distribution quotidienne (pendant six semaines) de $100 \mathrm{~g}$ d'un régime contenant 800 ppm de gossypol libre à des coqs de souche chair entraîne une augmentation significative de la proportion de spermatozoïdes malformés dans les éjaculats (17,6 p. 100 contre 10,6 p. 100 
TABLEAU IV Essai 1. Effet du gossypol (400 ppm) sur la qualité du sperme.

\begin{tabular}{|l|c|c|c|}
\hline Régime des coqs & Témoin & $\begin{array}{c}\text { Gossypol } \\
(400 \mathrm{ppm})\end{array}$ & SEM \\
\hline Poids vif $(\mathrm{g})$ & $3492 \mathrm{a}$ & $3510 \mathrm{a}$ & 103 \\
\hline $\begin{array}{l}\text { Consommation } \\
\text { alimentaire }(\mathrm{g} / \mathrm{j})\end{array}$ & 115 & 115 & - \\
\hline $\begin{array}{l}\text { Consommation de } \\
\text { gossypol (mg/kg/j) }\end{array}$ & - & 13,10 & - \\
\hline Motilité & $5,13 \pm 0,5 \mathrm{a}$ & $5,37 \pm 0,12 \mathrm{a}$ & 0,16 \\
\hline $\begin{array}{l}\text { Concentration } \\
\left(\times 10^{9} \text { Spz } / \mathrm{ml}\right)\end{array}$ & $6,92 \pm 0,55 \mathrm{a}$ & $8,12 \pm 0,54 \mathrm{~b}$ & 0,25 \\
\hline Vol. éjaculat $(\mathrm{ml})$ & $0,18 \pm 0,03 \mathrm{a}$ & $0,20 \pm 0,05 \mathrm{a}$ & 0,02 \\
\hline
\end{tabular}

TABLEAU V Essai 2. Effet du gossypol (800 ppm) sur la qualité du sperme.

\begin{tabular}{|l|c|c|c|}
\hline Régime des coqs & Témoin & $\begin{array}{c}\text { Gossypol } \\
(800 \mathrm{ppm})\end{array}$ & SEM \\
\hline $\begin{array}{l}\text { Poids d'un } \\
\text { éjaculat }(\mathrm{g})\end{array}$ & $0,63 \pm 0,34 \mathrm{a}$ & $0,77 \pm 0,23 \mathrm{a}$ & 0,13 \\
\hline $\begin{array}{l}\text { Concentration } \\
\times 10^{9} \text { Spz/ml) }\end{array}$ & $5,47 \pm 1,88 \mathrm{a}$ & $5,91 \pm 0,1 \mathrm{a}$ & 0,64 \\
\hline Motilité & $4,6 \pm 2,3 \mathrm{a}$ & $4,2 \pm 1,4 \mathrm{a}$ & 0,845 \\
\hline $\begin{array}{l}\text { Spermatozoïdes } \\
\text { morts (p. 100) }\end{array}$ & $8,60 \mathrm{a}$ & $9,2 \mathrm{a}$ & 1,03 \\
\hline $\begin{array}{l}\text { Spermatozoïdes } \\
\text { malformés (p. 100) }\end{array}$ & $10,61 \mathrm{a}$ & $17,36 \mathrm{~b}$ & 1,22 \\
\hline $\begin{array}{l}\text { Spermatozoïdes } \\
\text { morphologiquement } \\
\text { normaux }\end{array}$ & $80,79 \mathrm{a}$ & $73,35 \mathrm{~b}$ & 2,93 \\
\hline
\end{tabular}

$(a, b)$ : Les valeurs sur une même ligne suivies de la même lettre ne sont pas significativement différentes entre elles (seuil : 5 p. 100).

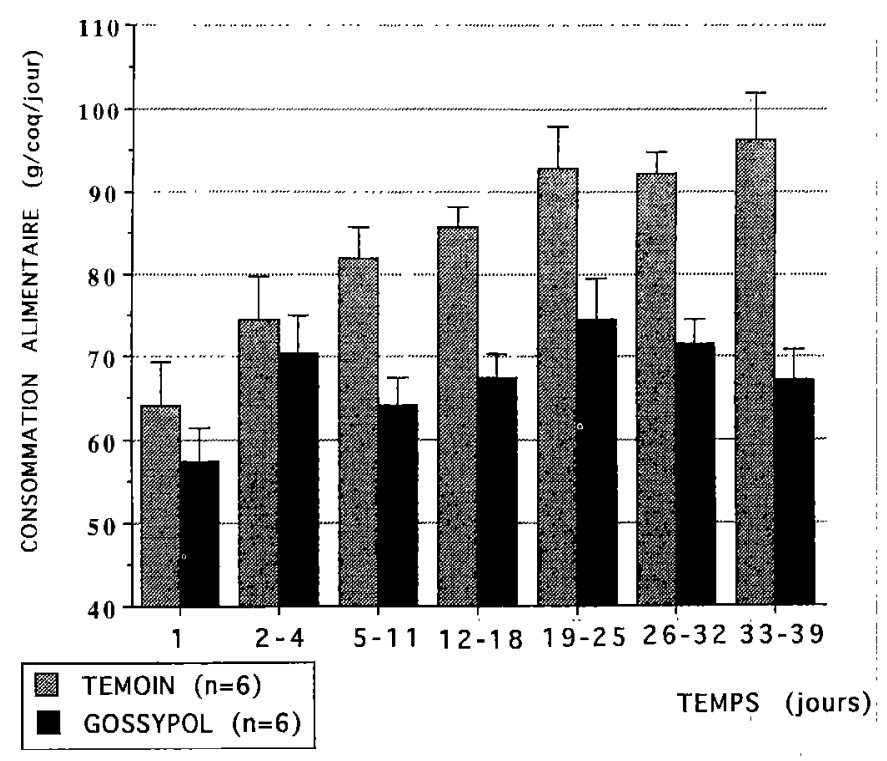

Figure 1 : Essai 3 : Consommation alimentaire moyenne (et écart-type de la moyenne) de coqs de souche ponte, recevant des régimes contenant 800 ppm de gossypol ou non

Toutefois, au plan zootechnique (tableau VI), les coqs consommant du gossypol ont une tendance (non significative) à moins consommer d'aliment (malgré le rationnement) que les témoins. La différence de consommation est de $3,4 \mathrm{~g} / \mathrm{j}$ entre les deux traitements, elle induit une diminution de poids vif de $2,3 \mathrm{~g} / \mathrm{j}$, chez les coqs traités par rapport aux coqs témoins. Les poids moyens du foie et de la rate des coqs ayant consommé du gossypol est significativement $(P<0,05)$ supérieur à ceux des coqs témoins. Les poids des testicules et du cœur ne sont pas modifiés significativement. On note une couleur anormale cuivrée verdâtre des foies chez les coqs traités qui permet de différencier très nettement les coqs ayant consommé du gossypol de ceux ayant reçu les rations témoins.

\section{Essai 3}

\section{Effets de 800 ppm de gossypol sur la consommation alimentaire de coqs reproducteurs ponte nourris ad libitum}

Après une augmentation progressive de l'ingéré quotidien des animaux témoins pendant les deux premières semaines du test due à leur adaptation aux mangeoires individuelles, les jeunes coqs de souche ponte réduisent rapidement leur ingéré alimentaire (figure 1) lorsque l'aliment contient 800 ppm de gossypol. La différence entre les deux traitements est significative dès la seconde semaine de test $(P<0,05)$. Pendant les 7 semaines d'expérience la réduction moyenne de consommation alimentaire est de 22 p. 100 en valeur relative par rapport 
TABLEAU VI Essai 2 et 3 . Effet de 800 ppm de gossypol dans le régime des coqs reproducteurs de souches chair ou ponte sur la consommation alimentaire, le poids vif et le poids de différents organes.

\begin{tabular}{|c|c|c|c|}
\hline & Témoin & $\begin{array}{l}\text { Gossypol } \\
(800 \text { ppm) }\end{array}$ & SEM \\
\hline \multicolumn{4}{|c|}{$\begin{array}{l}\text { Essai } 2 \text { : coqs « chair » } \\
\text { (durée de l'expérience : } 6 \text { semaines) }\end{array}$} \\
\hline Effectif & 5 & 5 & \\
\hline Poids vif $(g)$ & $4016 \pm 239 a$ & $3821 \pm 177 a$ & 86 \\
\hline $\begin{array}{l}\text { Consommation } \\
\text { alimentaire }(\mathrm{g} / \mathrm{j})\end{array}$ & $97,4 \pm 1,8 a$ & $94,0 \pm 3,2 a$ & 1,06 \\
\hline $\begin{array}{l}\text { Consommation } \\
\text { de gossypol } \\
\text { (mg/kg/jour) }\end{array}$ & - & $19,4 \pm 1$ & \\
\hline Gain de poids (g) & $26,8 \pm 111,7 a$ & $-69 \pm 38,95 a$ & 37,4 \\
\hline $\begin{array}{l}\text { Poids des organes }(g) \\
\text { foie } \\
\text { testicules } \\
\text { cceur } \\
\text { rate }\end{array}$ & $\begin{array}{c}31,3 \pm 1,8 \mathrm{a} \\
34,2 \pm 7 \mathrm{a} \\
15,4 \pm 3 \mathrm{a} \\
2,5 \pm 0,6 \mathrm{a}\end{array}$ & $\begin{array}{r}35,0 \pm 2 b \\
40,5 \pm 8,6 a \\
14,1 \pm 1,9 a \\
3,7 \pm 0,6 b\end{array}$ & $\begin{array}{l}0,86 \\
3,64 \\
0,96 \\
0,26\end{array}$ \\
\hline
\end{tabular}

Essai 3 : coqs « ponte »

(durée de l'expérience : 7 semaines)

\begin{tabular}{|c|c|c|c|}
\hline Effectif & 6 & 6 & \\
\hline Poids vif $(g)$ & $2807 \pm 323 a$ & $2537 \pm 175 b$ & 106 \\
\hline $\begin{array}{l}\text { Consommation } \\
\text { alimentaire }(g / j)\end{array}$ & $88,8 \pm 8,8 a$ & $69 \pm 7,9 b$ & 3,43 \\
\hline $\begin{array}{l}\text { Consommation de } \\
\text { gossypol } \\
(\mathrm{mg} / \mathrm{kg} / \mathrm{j})\end{array}$ & - & $21,2 \pm 1,7$ & - \\
\hline Gain de poids $(\mathrm{g})$ & $152,7 \pm 76,9 a$ & $-40,7 \pm 36,66 b$ & 24,59 \\
\hline $\begin{array}{l}\text { Poids des organes }(g) \\
\text { foie } \\
\text { testicules } \\
\text { cœur } \\
\text { rate } \\
\text { reins } \\
\text { gras abdominal }\end{array}$ & \begin{tabular}{|c|}
$30,2 \pm 5,8 \mathrm{a}$ \\
$28,1 \pm 3,9 \mathrm{a}$ \\
$17,3 \pm 2,6 \mathrm{a}$ \\
$3,2 \pm 0,7 \mathrm{a}$ \\
$11,6 \pm 1,38 \mathrm{a}$ \\
$34,02+27,5 \mathrm{a}$
\end{tabular} & $\begin{array}{c}27,4 \pm 4,9 a \\
21,5 \pm 3,7 b \\
14,4 \pm 2,1 \mathrm{a} \\
3,1 \pm 0,32 \mathrm{a} \\
11,5 \pm 2,9 \mathrm{a} \\
4,6 \pm 3,2 \mathrm{~b}\end{array}$ & $\begin{array}{l}2,45 \\
1,73 \\
1,08 \\
0,25 \\
1,06 \\
8,95\end{array}$ \\
\hline
\end{tabular}

$(a, b)$ : Les valeurs sur une même ligne suivis de la même lettre ne sont pas significativement différentes entre elles (seuil : 5 p. 100). aux témoins soit près de $20 \mathrm{~g}$ par jour et par coq (tableau VI). Cette variation s'accompagne d'une diminution simultanée de la prise de poids d'environ: $200 \mathrm{~g}$ (soit 3,9 g/j de traitement). On observe également lune variation notable du poids de gras abdominal moyen ( $34 \mathrm{~g} / \mathrm{coq}$ pour les animaux témoins et seulement $4,6 \mathrm{~g} / \mathrm{coq}$ pour les animaux consommant du gossypol). Cette différence est significative au seuil de probabilité de 95 p. 100 malgré une variabilité individuelle élevée sur ce critère. De la même manière on observe un poids moyen des testicules plus faible chez les coqs ayant consommé du gossypol $(21,5 \mathrm{~g})$ par rapport aux témoins $(28,1 \mathrm{~g} ; \mathrm{P}<0,05)$ à opposer à la tendance NS de l'essai 2.

Comme dans l'essai 2, l'aspect macroscopique des foies des coqs traités diffère nettement en couleur de celui des foies des coqs témoins. La couleur des organes provenant des coqs ayant consommé du gossypol est brun verdâtre et suggère une rétention biliaire. Cependant, les résultats d'analyse histologique n'ont révélé aucune lésion particulière tant au niveau des foies qu'à celui des testicules ou de la rate.

\section{DISCUSSION}

Le gossypol extrait de soapstock de coton, ajouté sous la forme d'acétate aux concentrations de 250 et 400 ppm de gossypol libre à des aliments équilibrés, distribués à des coqs et des poules pubères, pendant respectivement 9 et 7 semaines, n'a affecté aucune des performances de reproduction mesurées dans nos conditions d'expérience (milieu tropical). Ce résultat est en désaccord avec les travaux de nombreux auteurs dont WALDROUP (25), BERARDI et GOLDBLATT (5), selon lesquels le gossypol diminuerait les capacités de reproduction des coqs et poules lorsqu'il est présent à des concentrations inférieures à 200 ppm. Il faut cependant remarquer que la plupart de ces expériences étudient des régimes à base de tourteau de coton dont la teneur en gossypol a été calculée à partir de celle du tourteau.

Les nombreuses réactions de liaison du gossypol avec d'autres composants du tourteau de coton (protéines, glucides) sont catalysées au cours du chauffage $(4,9)$. Les produits de ces réactions pourraient être éventuellement responsables d'une partie des effets, non reproduits dans ces essais en l'absence' de tourteau de coton entier. On confirme ainsi plusieurs travaux sur poulets de chair $(7,11,17)$ qui ne rapportent aucun effet du gossypol à des concentrations alimentaires égales ou supérieures à 400 ppm. D'autre part NARAIN et al., en 1960 (18), constatent qu'un régime à concentration élevée en protéines peut compenser les effets négatifs du gossypol. Dans les travaux des auteurs, l'équilibre nutritionnel des aliments testés dans l'essai 1 , pourrait avoir limité les effets éventuels du gossypol ajouté. Bien que la durée 
T. Dongmo, M. Pouilles-Duplaix, M. Picard, C. Mbi, M. De Reviers

limitée de l'expérience (9 semaines) d'une part et la productivité relativement faible des poules d'autre part ( $44 \mathrm{~g}$ d'œuf par jour) puissent avoir partiellement masqué l'effet du gossypol, les résultats de fertilité (>90 p. 100) obtenus à partir de 1522 œufs, suggèrent que des rations alimentaires équilibrées contenant 400 ppm de gossypol n'ont que peu ou pas d'action sur la reproduction. Pour une concentration supérieure (800 ppm) le gossypol réduit significativement la consommation alimentaire des coqs de souche ponte mais pas celle des coqs de souche chair. Huit cents ppm correspondent à environ $20 \mathrm{mg} / \mathrm{kg}$ de poids vif $/ \mathrm{j}$ et les effets observés sont comparables à ceux publiés en 1985 par AKANBI (2) qui administre des doses de 0, 20, 40 et $80 \mathrm{mg} / \mathrm{kg}$ de gossypol à des coqs "White Leghorn" pendant 42 jours. Des concentrations aussi élevées ne correspondent pas à la situation nutritionnelle induite par l'utilisation de tourteau de coton comme source principale de protéines dans l'alimentation des volailles, mais se rapprochent plutôt des doses expérimentales utilisées chez certains mammifères $(13,15)$. L'action de doses élevées de gossypol sur le poids des testicules (8) ou celui des ovaires (1) ou encore sur la proportion de spermatozoïdes malformés (essai 2) peut être due à une action directe de cette substance sur les organes génitaux (les cellules germinales bénéficient pourtant de nombreuses protections tissulaires) ou bien des dysfonctionnements métaboliques induits par une intoxication par le gossypol. Celle-ci se traduit dans l'essai 2 par une augmentation du poids du foie et de la rate ct dans l'essai 3 par une forte coloration hépatique et une réduction importante de l'ingéré alimentaire.

II faut cependant faire remarquer que le régime alimentaire distribué aux coqs au cours de l'ossai 3 est un aliment de ponte riche en calcium (3,5 p. 100). Cette concentration nettement supérieure aux besoins de l'animal pourrait éventuellement accentuer les effets du gossypol sur la réduction de l'ingéré. Ce dernier point constitue une hypothèse à vérifier compte tenu de la fréquente utilisation pratique des régimes de ponte dans l'alimentation des coqs reproducteurs.

Le dosage précis des résidus de gossypol dans les testicules, le sperme ou les ovaires et l'étude du mode d'action du gossypol sur les spermatozoïdes conservés in vitro devraient permettre de progresser dans la compréhension des mécanismes impliqués.

En résumé, les résultats des expériences rapportées dans ce travail et ceux de DONGMO et al. (10), ne soutiennent pas l'hypothèse d'une toxicité alimentaire du tourteau de coton sur la reproduction des volailles attribuable en totalité au gossypol libre, au moins pour les doses habituellement rencontrées dans cette matière première.

\section{CONCLUSIONS}

L'extraction et la purification de $650 \mathrm{~g}$ d'acétate de gossypol à partir de soapstock de coton ont permis d'étudier les effets de cette molécule sur la reproduction des volailles, en l'incorporant directement à des régimes équilibrés ne contenant pas de tourteau de coton.

Dans ces conditions, une concentration alimentaire de 400 ppm de gossypol libre n'a pas affecté les capacités de reproduction des coqs et des poules après respectivement 9 et 7 semaines de distribution (il faut rappeler ici que 400 ppm de gossypol libre correspond à une concentration bien supérieure à l'apport maximum possible du tourteau de coton dans un aliment pour volaille). Les effets du tourteau de coton sur la reproduction sont donc probablement imputables à d'autres facteurs que le gossypol libre à moins qu'ils ne révèlent des interactions entre cette molécule et d'autres nutriments.

A la concentration alimentaire de $800 \mathrm{ppm}$ (ou $20 \mathrm{mg} / \mathrm{kg}$ $\mathrm{PV} / \mathrm{i}$ ) le gossypol étudié déprime l'ingéré alimentaire et le poids des coqs adultes de souche ponte après 6 à 7 semaines de distribution, tout en modifiant l'aspect macroscopique du foie. Dans ces conditions, le poids des testicules est réduit (sans altération histologique identifiable), et la proportion de spermatozoïdes anormaux par éjaculat augmente. Le mode d'action direct ou indirect du gossypol à ce niveau est discuté.

\section{REMERCIEMENTS}

Les auteurs expriment leur gratitude au ministre français de la Coopération, à l'Institut de recherches zootechniques et vétérinaires du Cameroun et à l'INRA de Nouzilly pour le financement de ces travaux. Nos remerciements vont également à $M$. Patrice LEFÈVRE (CIRAD-EMVT), Mme MARQUIE (CIRAD-CA) pour leur contribution à la mise au point de la méthode d'extraction du gossypol, aux Drs NGOU NGOUPAYOU, BLESBOIS, BRILLARD et LETERRIER pour leurs conseils scientifiques. Enfin nous sommes reconnaissants à $M$. Siméon NGANWA, Mme TCHAKOUNTÉ, MM. PROCHORE, MESLIER et GAULTHIER pour leur assistance technique. 


\section{BIBLIOGRAPHIE}

1. AHMED (A.A.), SOLIMAN (M.M.), YOUNIS (M.), ZAKI (A.), KHALIFA (B.A.A.). Gossypol as antifertility agent in female rats. Arch. exp. VetMed., 1988, 42 : 944-948.

2. AKANBI (O.C.). Reproductive effects of gossypol and cottonseed meal in male single comb White Leghorn chickens. Diss. Abstr. int.B , $1985, \mathbf{4 5}$ (7) $: 1977$

3. AKANBI (O.), NAKAVE (H.S.), ARSCOTT (G.H.). Addition of gossypol acetic acid in the feed on reproductive traits and androgen level of single comb White Leghorn (SCWL) roosters.Poult. Sci., $1984 ; 65-51$ (abst.).

4. ALTSCHUL (A.M.). Processed plant protein foodstuffs. New York, Academic Press Inc. Publ., 1958. 955 p.

5. BERARDI (L.C.), GOLDBLATT (L.A.). Gossypol. In: LIENER (I.E.) Ed. 'loxic constituents of plant foodstuffs. 2nd ed. New York, Academic Press, 1980. p. 183-237.

6. BURROWS (W.H)., QUINN (J.P.). The collection of spermatozoa from the domestic fowl and turkey. Poult. Sci., 1937, 16 : 19-24.

7. COUCH (J.R.), CHANG (W.Y.), LYMAN (C.M.). The eflect of free gossypol on chick growth. Poult. Sci., 1955, 34 : 178-183.

8. COULSON (P.B.), SNELL (R.L.), PARISE (C.). Short term metabolic effects of the antifertility agent, gossypol on various reproductive organ of male mice. Int. J. Andr. , 1980, 3 : 507-518.

9. DECHARY (J.M.), KUPPERMAN (R.P.), THURBER (F.H.), ALTSCHUL (A.M.). Removal of gossypol from cottonseed by solvent extraction procedures. J. Am. Oil Chom. Soc., 1952: 339-341.

10. DONGMO (T.), POUILLES-DUPLAIX (M.), NGOU-NGOUPAYOU (J.D.), BLESBOIS (E.), DE REVIERS (M.). Utilisation du tourtcau de coton dans l'alimentation des volailles. 1. Etude zootechnique chez les reproducteurs de l'espèce Gallus dontesticus. Revue Élev. Méd. vét. Pays trop., 1993, 46 (1) : 609-619.

11. EWING (W.R.). Poultry nutrition. 5th ed. Pasadena, USA, The Ray Ewing Company, Division of Hoffman-Laroche. 1963. 1475 p.

12. GIRIDHARAN (N.), SESIKERAN (B.), BAMJI, MAHTAB. MAD HYASTHA (M.N.). Dose and time related changes in LDH-X activity, epidymal carnitine levels and fertility in gossypol treated male rates. Contraception, 1987, 35: 89-100.

13. JENSEN (D.R.), TONE (J.N.), SORENSEN ( R.H.). BOZEK (S.A.) Deposition pattern of the antifertility agent, gossypol in selected organs of male rats. Toxicology, $198224: 65-72$.
14. KALLA (N.R.), FOO (J.T.W.), SHETH (A.R.). Studies on the male antifertility agent gossypol acetic acid. V. Effect of gossypol acetic acid on the fertility of male rats. Andrologia, 1982, 14 (6) : 492-500.

15. LAGERLÖF (K.R.),TONE (N.J.). The effect of gossypol acetic acid on female reproduction. Drug Chem. Toxic., 1985, 8 (6) : 469-482.

16. LAKE (P.E.), STEWART (J.M.). Artificial insemination in poultry. London. Ministry of Agriculture, Fisheries and Food, Her Majesty's Stationary office, 1978. (Bulletin 213)

17. NARAIN (R.), LYMAN (C.M.), COUCH (J.R.). High levels of free gossypol in hen diets : effects on body weight.' teed consumption, and egg production. Poult. Sci, 1957, 36: 1351-1354.

18. NARAIN (R.), LYMAN (C.M.). DEYOE (C.W.), COUCH (J.R.). Effect of protein level of the diet on free gossypol tolerance in chicks. Poult. Sci.., 1960, 39: 1556-1559.

19. PETITJEAN (M.J.). Recherches sur l'estimation du pouvoir fécondant des coqs. Poitiers, Mémoire d'Ingénieur du CNAM, 1965. 84 p.

20. PHELPS (R.A.). Cottonseed meal for poultry : from research to practical application. Wld's Poult. Sci. J., 1966, 22: 86-112.

21. PONS (W.A.), POMINSKI (J.), KING (W:H.), HARRIS (J.A.), HOPPER (T.H.). Recovery of gossypol from cottonseed gums. J. Am. Oil Chem. Soc., 1959, $36: 328-332$.

22. ROJAS (S.W.), SCOTT (M.L.). Factors affecting the nutritive value of cotton seed meal as a protein source in ducks diets. Poult. Sci.., 1969, 48 : $818-835$.

23. SAVII LE (D.G.), SMITH (I.), NICHOI LS (P.). Cottonseed meal as a source of protein in diets for laying hens. Aust. J. exp. Agric. Anim. Husb., $1969,9: 413-416$.

24. SCOTT (H.M.), MUELLER (D.N.). The porosity of the egg shell in relation to hatchability. Poult. Sci., 1940, $19: 163-166$

25. WALDROUP (P.W.). Cottonseed meal in poultry diets. Feedstuffs ,1981, 53: 21-24.

26. WHITE (I.G.), VISHWANATH (R.), SWAN (M.A.), WOODMAN (B.P.D.). Studies of the echanism of action of gossypol as a male antifertility agent. Contraception, 1988, 37. (3): 269-277.

27. YANG (Y.G.), WU (X.Y.). Antifertility mechanisms of gossypol acetic acid in females rats. J. Reprod. Fert. . 1987, 80:425-429.

28. YO (T.). Utilisation directe des graines de coton décortiquées de variétés sans gossypol dans l'alimentation des poulets de chair en Côte-d'Ivoire. Revue Élev. Méd. vét. Pays trop. . 1991, 44 (3) : 355-360. 


\section{T. Dongmo, M. Pouilles-Duplaix, M. Picard, C. Mbi, M. De Reviers}

DONGMO (T.), POUILLES-DUPLAIX (M.), PICARD (M.), MBI (C.), DE REVIERS (M.). Use of cottonseed oil-meal in poultry feeding. II. Effects of gossypol on the reproductive parameters. Revue Elev. Méd. vét. Pays trop., $1993, \mathbf{4 6}$ (4) : 621-630

Three experiments were carried out to determine the effects of gossypol, administred via the diet, on the reproductive performances of adult cocks and hens of egg-laying or broiler strains. A total of $650 \mathrm{~g}$ gossypol acetate were extracted from $80 \mathrm{~kg}$ cotton soapstock and cristallised. The product obtained included $47.86 \%$ free gossypol and $61.19 \%$ total gossypol. Balanced diets without cottonseed oilmeal were supplemented with increasing amounts of free gossypol $(0$, $250,400,800 \mathrm{ppm}$ ) and offered to breeding cocks and hens. The first trial was made with adult hens and cocks ( 128 and 45 , respectively). It did not show any effect of using dietary gossypol amounts lower or equal to $400 \mathrm{ppm}$ on feed intake, egg-laying, semen production and motility, fertility and hatchability of incubated eggs. The two following trials were performed to determine the effect of $800 \mathrm{ppm}$ of dietary frec gossypol on the performances of ten 82-week old breeding cocks of the broiler strain subjected to feed restriction and on twelve 29-week old breeding cocks of the laying strain fed ad libitum This amount of free gossypol (about $20 \mathrm{mg} / \mathrm{kg}$ liveweight/day) reduced the feed intake and liveweight of the animals as well as the testicle weight of cocks of the laying strain and it increased the proportion of abnormal spermatozoa in the ejaculates of cocks of the broiler strain. In addition, the liver of cocks fed gossypol supplemented diets exhibited an abnormal brown-green colour. However, on account of the low gossypol concentrations of cottonseed oil-meal $(<250 \mathrm{ppm})$, the free gossypol contained in this meal is most likely not responsible for the reproductive disorders observed in poultry fed with this kind of feed.

Key words : Poultry - Cock - Layer hen - Broiler - Feeding - Cottonseed cake- Gossypol - Reproduction - Laying performance - Egg hatchability Sperm - Weight.
DONGMO (T.), POUILLES-DUPLAIX (M.), PICARD (M.), MBI (C.), DE REVIERS (M.). Uso de 'la torta de algodón en la alimentación aviar. II. Efectos del gosipol sobre los parámetros reproductivos. Revue Élev. Méd. vét. Pays trop, 1993, 46 (4) : 621-630

Los efectos del gosipol administrado por vía oral fueron medidos mediante tres experiencias, llevadas a cabo en reproductores adultos, hembras y machos, de postura o de carne. A partir de $80 \mathrm{~kg}$ de "soapstock" de algodón, se extrajeron y cristalizaron $650 \mathrm{~g}$ de acetato de gosipol. El producto final presentó un título de 47,86 p. $100 \mathrm{de}$ gosipol libre ye 61,19 p. 100 de gosipol total. Los alimentos equilibrados, desprovistos de torta de algodón, fueron complementados con dosis crecientes de $0,250,400$ y $800 \mathrm{ppm}$ de gosipol libre y distribuídos a gallos y gallinas reproductoras. EI primer ensayo se llevó a cabo sobre 128 gallinas y 45 gallos púberes. No se demostró ningún efecto con dosis alimenticias inferiores o iguales a $400 \mathrm{ppm}$ de gosipol libre, sobre el consumo alimenticio, la postura, la producción y la motilidad espermáticas, la fecundidad o la tasa de eclosión de los huevos incubados. Los dos ensayos siguientes sirvieron para probar el efecto de una concentración alimenticia de $800 \mathrm{ppm}$ de gosipol libre sobre 10 gallos reproductores de carne, de 82 semanas y con dietas racionadas, y doce gallos reproductores de postura, de 29 semanas y alimentados ad libitum. A esta dosis (alrededor $20 \mathrm{mg} / \mathrm{kg}$ PV/día), el gosipol libre disminuyó el consumo alimenticio, el peso de los animales y de los testículos de los machos de cepa postura e hizo aumentar la proporción de espermatozoides anormales en los eyaculados de gallos de carne. Con esta concentración, se observó un color anormal, café verdoso, en los hígados de los gallos que consumieron las dietas complementadas con gosipol. Dadas las concentraciones de gosipol en la torta de algodón (generalmente inferiores a $250 \mathrm{ppm}$ ), parece poco probable que el gosipol libre de esta torta sea el responsable directo de los disturbios reproductivos observados en las aves que lo consumen.

Palabras claves : Aves de corral - Gallo - Gallina ponedora - Pollo de engorde - Alimentación - Torta de algodón - Gosipol - Reproducción Rendimiento en la postura - Eclosabilidad del huevo - Esperma - Peso. 